\title{
La proyección exterior de la identidad europea: Política Comercial Común y condicionalidad en materia de Derechos Humanos*
}

\author{
The external projection of the european identity: Common Commercial \\ Policy and conditionality in the matter of Human Rights
}

\author{
Maria Julià Barceló \\ Profesora de Derecho Internacional Público y de la UE de la Universitat Oberta de Catalunya \\ mjuliabar@uoc.edu
}

doi: http://dx.doi.org/10.18543/ced-02-2019pp287-308

\begin{abstract}
Sumario: I. Introducción.-II. Proyección exterior de la identidad europea: Marco estratégico de derechos humanos y democracia. 1. Primer plan de acción (2012-2014). 2. Segundo plan de acción (2015-2019)._-III. Política comercial común y condicionalidad. 1 . Origen y tipología de las cláusulas de condicionalidad. 2. La cláusula democrática y de derechos humanos. 3. Otras formulaciones. 4. Efectos jurídicos de la condicionalidad.-IV. Instrumentos comerciales unilaterales y condicionalidad. 1. La condicionalidad social y el nuevo sistema de preferencias generalizadas. 2. Régimen general y régimen específico. 3. Procedimiento de seguimiento. 4. Práctica de la Unión Europea.-V. Reflexiones finales.
\end{abstract}

Resumen: Uno de los aspectos distintivos de la actual Unión Europea - más allá de su concepción como organización económica - es la protección y promoción de los derechos humanos en su actuación exterior. En aplicación del mandato establecido en el TUE, en sus relaciones con el resto del mundo, la Unión contribuye a la protección de los derechos humanos, así como al respeto y desarrollo del derecho internacional a través de la ejecución de políticas comunes. El Marco estratégico sobre derechos humanos y democracia establece los principios que prevén la integración de los derechos humanos en todas las políticas de la Unión. La política comercial común es uno de los ejes de esta actuación, en particular a través de dos instrumentos: la cláusula de derechos humanos y democracia-incorporada en los acuerdos comerciales - y el Sistema de Preferencias Generalizadas - otorgado a los países en vías de desarrollo-. La base de estos instrumentos es la condicionalidad, mayoritariamente positiva, destinada a ganar la confianza de los países terceros e incorporarlos al comercio para mejorar sus economías. Aún así, estos instrumentos adolecen de ciertos problemas técnicos-jurídicos y políticos,

* Recibido el 2 de julio de 2018, aceptado el 5 de septiembre de 2018.

Cuadernos Europeos de Deusto 
que pueden dificultar una actuación transparente y sin arbitrariedades de la Unión respecto a la protección de los derechos humanos.

Palabras clave: identidad exterior europea, política comercial común, cláusula de derechos humanos y democracia, sistema de preferencias generalizadas, condicionalidad.

Abstract: One of the distinctive aspects of the current EU -beyond its conception as an economic organization- is the protection and promotion of human rights in its external action. In application of the mandate established in the TEU, in its relations with the rest of the world, the Union contributes to the protection of human rights, as well as the respect and development of international law through the execution of common policies. The Strategic Framework on Human Rights and Democracy establishes the principles that provide for the integration of human rights in all policies of the Union. The common commercial policy is one of the axes of this action, in particular through two instruments: the clause on human rights and democracy -incorporated in trade agreements-and the Generalised system of preferences - granted to developing countries-. The basis of these instruments is conditionality, majoritarian positive, aimed at gaining the confidence of third countries and incorporating them into trade to improve their economies. Even so, these instruments suffer from certain technical-legal and political problems, which may hinder the transparent and non-arbitrary action of the Union with respect to the protection of human rights.

Keywords: European External Edentity, common commercial policy, human rights and democracy clause, Generalised system of preferences, conditionality.

\section{Introducción}

Uno de los aspectos distintivos de la Unión Europea (UE) que influyen en la configuración de su identidad, más allá de su concepción como una organización de carácter puramente económico, es la protección y promoción de los derechos humanos a través de su acción exterior, tal y como recoge el art. 2 del Tratado de la Unión Europea (TUE), al establecer el respeto a los derechos humanos como punto de partida para la actuación de la UE. Es bien sabido también, que el desarrollo de la identidad europea se ha proyectado tanto a nivel interior $(\text { ad intra })^{1}$ como exterior

1 Véase por ejemplo, Laura Huici Sancho, «Austeridad y crisis: La responsabilidad de la Unión Europea y sus Estados miembros en la toma de decisiones que repercuten en el goce y disfrute de los derechos económicos, sociales y culturales» en La exigibilidad de los derechos económicos, sociales y culturales en la Sociedad internacional del siglo XXI: una aproximación jurídica desde el Derecho internacional, ed. por Jordi Bonet Pérez y Rosa Ana Alija Fernández (Barcelona: Marcial Pons, 2016), 373-398. 
$(\text { ad extra })^{2}$ de la Unión. En este sentido, los artículos 55, 56 y 103 de la Carta de las Naciones Unidas establecen la primacía del Derecho Internacional de los derechos humanos sobre cualquier otro tipo de obligación internacional. La prevalencia de las obligaciones de la Carta ante un conflicto entre las obligaciones contraídas por los Estados miembros de Naciones Unidas y las contraídas en virtud de cualquier otro convenio internacional debe ser aplicada y respetada también en la celebración de los tratados internacionales cuyo objeto es el comercio internacional. Naciones Unidas aludía en la Agenda 2030 para el Desarrollo Sostenible al comercio internacional y a su impulso como instrumento para promover la reducción de la pobreza y el fomento del desarrollo sostenible, cuestión que indudablemente actúa en beneficio de los derechos humanos ${ }^{3}$. En este contexto, la primacía del derecho internacional de los derechos humanos también vincula a la Unión Europa y a sus Estados miembros en sus relaciones con el exterior.

A lo largo de los años Europa ha configurado su identidad a través de una tipología diversa de instrumentos normativos que se refieren a una tradición europea y, en cierto modo, a una teoría de los derechos europea. Entre los elementos de esta identidad se incluyen, entre otros, los principios de la democracia representativa, el imperio de la ley y el respeto de los derechos humanos. El preámbulo del Tratado de Lisboa (2007) se remite a «la herencia cultural, religiosa y humanista de Europa, a partir de la cual se han desarrollado los valores universales de los derechos inviolables e inalienables de la persona, así como la libertad, la democracia, la igualdad y el Estado de Derecho». Igualmente la Carta de derechos fundamentales de la UE se refiere a la dignidad humana, la libertad, la igualdad y la solidaridad, como fundamentos de la Unión, así como a los principios de la democracia y el Estado de Derecho. A partir de estas premisas parece posible hablar de una concepción europea de los derechos humanos, que va más allá de Europa y que se apoya en cuatro valores fundamentadores de los derechos: la dignidad humana, la libertad, la igualdad y la solidaridad. ${ }^{4}$

2 Véase por ejemplo, Maria Julià Barceló, «Los derechos económicos, sociales y culurales en las relaciones exteriores de la Unión Europea» en La exigibilidad de los derechos económicos, sociales y culturales en la Sociedad internacional del siglo XXI: una aproximación jurídica desde el Derecho internacional, ed. por Jordi Bonet Pérez y Rosa Ana Alija Fernández (Barcelona: Marcial Pons, 2016), 401-420.

${ }_{3}$ Resolución A/RES/30/1, «Transformar nuestro mundo: la Agenda 2030 para el Desarrollo Sostenible», de la Asamblea General, de 21 de octubre de 2015.

${ }^{4}$ Rafael de Asís, «La Unión Europea y la importancia de los derechos humanos» en La acción exterior de la UE en materia de derechos humanos, coord. por Ana Manero Salvador (Getafe: Instituto universitario de Estudios Internacionales y Europeos, 2014), 12. Acceso el 10 de enero de 2018, https://e-archivo.uc3m.es/bitstream/handle/10016/21126/ monografias_4_IFV_2014.pdf?sequence=1\&isAllowed=y. 
Incluso podemos considerar que en la actualidad esta identidad europea dispone de un sistema institucional a su servicio. La puesta en marcha del Servicio Europeo de Acción Exterior (SEAE) ha comportado la creación en su seno de una Dirección de derechos humanos y democracia. Igualmente, a través de la Alta Representante y el COHOM (Grupo de derechos humanos del Consejo ${ }^{5}$ ), así como del Representante especial de la UE en materia de derechos humanos, la Unión puede dar «visibilidad» y también «Voz» a la promoción y a la protección de los derechos humanos en sus actuaciones exteriores.

El objetivo de este trabajo es analizar la construcción de esta identidad europea exterior a través de la política comercial común. Y más concretamente, ver cómo los acuerdos de la política comercial de la Unión, y sus instrumentos comerciales unilaterales (en especial la cláusula de derechos humanos y democracia y el nuevo sistema de preferencias generalizas) intentan definir una identidad exterior de la UE, fundamentada en la condicionalidad. En primer lugar, situaremos la proyección exterior de la identidad europea a través del marco europeo actual de protección de los derechos humanos (apartado II). En segundo lugar, se analizará la política comercial de la Unión y las cláusulas de condicionalidad (apartado III). En tercer lugar, los instrumentos comerciales unilaterales de la UE que incluyen cláusulas de condicionalidad, con especial referencia al sistema de preferencias generalizadas (apartado IV) y, finalmente, se procederá a unas breves reflexiones finales sobre la condicionalidad en la política comercial común y la construcción de la identidad europea en materia de derechos humanos (apartado V).

\section{Proyección exterior de la identidad europea: Marco estratégico de derechos humanos y democracia}

El art. 3.5 del TUE establece que en sus relaciones con el resto del mundo la UE contribuirá a la protección de los derechos humanos, especialmente los derechos del niño, así como al respeto y desarrollo del derecho internacional, en particular los principios de la Carta de las Naciones Unidas ${ }^{6}$. A este fin, el art. 205 del Tratado de Funcionamiento de la Unión Eu-

${ }^{5} \mathrm{El} \mathrm{COHOM}$ define las prioridades estratégicas de la UE sobre cuestiones concretas de derechos humanos o geográficos. Acceso el 15 de enero de 2018, http://www.consilium.europa.eu/es/council-eu/preparatory-bodies/working-party-human-rights/).

6 Véase Antonio Blanc Altemir, «Las Naciones Unidas y la Unión Europea: valores y ámbitos de acción compartidos en aras de un multilateralismo más eficaz», en Las relaciones entre las Naciones Unidas y la Unión Europea. Seguridad, cooperación y derechos humanos, 
ropea (TFUE) dispone que la acción exterior de la Unión se sustente en los principios establecidos en el art. 21 del TUE, que dirigen a la Unión a definir y ejecutar políticas comunes y acciones destinadas, entre otras, a consolidar y respaldar la democracia, el Estado de Derecho, la universalidad e indivisibilidad de los derechos humanos y las libertades fundamentales y el respeto de la dignidad humanos. Con el objeto de desarrollar estas políticas comunes, el 25 de junio de 2012, el Consejo adoptó un Marco estratégico sobre derechos humanos y democracia ${ }^{7}$, en el que se definen los principios, objetivos y prioridades con vistas a mejorar la eficacia y coherencia de la política de la Unión durante los próximos diez años. Estos principios prevén la integración de los derechos humanos en todas las políticas de la Unión como «hilo conductor» también en los casos en que las políticas interiores y exteriores se superponen, así como la adopción de un enfoque mejor adaptado. A través de este Marco estratégico la UE adopta por primera vez un documento estratégico unificado. Anteriormente, todas las decisiones en materia de derechos humanos y democracia se contenían inicialmente en declaraciones que se centraban normalmente sobre determinados asuntos o países. Más adelante, la Unión pasó a elaborar una serie de líneas directrices y otras orientaciones sobre la línea de conducta que se debe seguir en este ámbito. Con este documento unificado, la UE demuestra su preocupación por los derechos humanos en toda su acción exterior y también el fomento de la universalidad de los derechos humanos, así como su voluntad de aplicar las prioridades de la UE en esta materia ${ }^{8}$. Para dotar de mayor eficacia y visibilidad a esta actuación exterior el 25 de julio de 2012, y a propuesta de la Alta Representante, se creó la figura del Representante especial de la UE para los derechos humanos ${ }^{9}$. La prórroga en febrero de 2017 de su mandato por dos años más da fe de la importancia y relevancia

dir. por Antonio Blanc Altemir (Madrid: Tecnos, 2013), 56. Y Joan David Janer Torrens, La protección de los derechos humanos y de los principios democráticos en las relaciones exteriores de la Unión Europea: mecanismos jurídicos, (Barcelona: Atelier, 2005). Más en general, José María Pérez de Nanclares, «The protection of human rights in the European Union», en International Human Rights Law in a Global Context, ed. por Fernando Gómez Isa y Koen de Feyter (Bilbao: University of Deusto, 2009) ,777-801.

7 Doc. 11417/12, «Derechos humanos y democracia: Marco estratégico y plan de acción de la UE», Bruselas, de 25 de junio de 2102. Acceso el 12 de febrero de 2018, https://eeas .europa.eu/sites/eeas/files/st_11855_2012_init_es.pdf .

${ }^{8}$ Doc. 11737/12, «La UE adopta un Marco estratégico sobre derechos humanos y democracia», Luxemburgo, 25 de junio de 2012:2. Acceso el 12 de febrero de 2018, http://www. consilium.europa.eu/uedocs/cms_data/docs/pressdata/ES/foraff/131507.pdf .

9 Decisión (PESC) 2012/440 del Consejo, de 25 de julio de 2012, por la que se nombra al Representante Especial de la Unión Europea para los Derechos Humanos (DOUE L 200 de 27 de julio de 2012). 
que ha ido adquiriendo esta figura en su interacción con los socios exteriores de la $\mathrm{UE}^{10}$.

\section{Primer plan de acción (2012-2014)}

El Marco estratégico iba acompañado de un Plan de Acción para su aplicación entre 2012 y 2014, estableciéndose las medidas específicas a aplicar por la Unión hasta el 31 de diciembre de 2014. En este sentido, las Directrices de la UE sobre derechos humanos adoptadas por el Consejo facilitan, pese a no ser jurídicamente vinculantes, instrucciones prácticas acerca de: a) la lucha contra la pena de muerte; b) los diálogos sobre derechos humanos; c) los derechos del niño; d) la lucha contra la tortura y otros tratos crueles; e) la protección de los niños en los conflictos armados; f) la protección de los defensores de los derechos humanos; g) el cumplimiento del Derecho internacional humanitario; y h) la lucha contra la violencia ejercida contra mujeres y niñas. Igualmente, se prevén directrices en materia de libertad de religión o de creencias, de los derechos de LGBT (lesbianas, gays, bisexuales y transexuales) y de la libertad de expresión, en y fuera de Internet ${ }^{11}$.

\section{Segundo plan de acción (2015-2019)}

Evaluado el primer Plan y siguiendo la orientación política de la Alta representante, el 20 de julio de 2015 el Consejo aprobó el segundo Plan de acción (2015-2019) ${ }^{12}$, con el objetivo de seguir en la línea marcada por el primero y fomentar los principios de no discriminación, igualdad de género y empoderamiento de la mujer, al tiempo que garantizar un enfoque global de la prevención y el abordaje de conflictos y crisis centrado en los derechos humanos. Estamos ante un plan continuista, que recoge el compromiso

10 Decisión (PESC) 2017/346 del Consejo, de 27 de febrero de 2017, por la que se prorroga el mandato del Representante Especial de la UE (DOUE L 50, de 28 de febrero de 2017).

11 Directrices Europeas de Derechos Humanos de la Delegación de la Union Europea en México, acceso el 12 de febrero de 2018, http://eeas.europa.eu/delegations/mexico/eu_ mexico/political_relations/human_rights/index_es.htm

12 «Comunicación conjunta al Parlamento Europeo y al Consejo. Plan de Acción sobre derechos humanos y democracia (2015-2019). Mantener los derechos humanos entre las prioridades de la agenda de la UE», Bruselas, 28.4.2015 JOIN (2015) 16 final. Acceso el 28 de diciembre de 2017, http://www.consilium.europa.eu/es/press/press-releases/2015/07/20/fachuman-rights/pdf 
de la UE de aplicar todo el programa de derechos humanos y democracia reflejado en el Marco estratégico de 2012, que sigue orientando su actuación. Todo ello en colaboración con países de todas las regiones, con las organizaciones internacionales y la sociedad civil. De los cinco ámbitos estratégicos de actuación que establece este plan, destaca el punto cuarto, en el que la UE manifiesta su voluntad de integrar en mayor medida los derechos humanos en los aspectos exteriores de sus políticas, a fin de dotar de una mayor coherencia sus políticas exteriores, entre ellas, el comercio ${ }^{13}$. El 16 de octubre de 2017 el Consejo adoptó unas Conclusiones sobre la revisión intermedia del Plan de Acción para los Derechos Humanos y la Democracia, en la que se comprometía a seguir la aplicación del plan de acción durante el 2019, destacando la necesidad de continuar en la línea de promoción de los derechos humanos la democracia y el imperio de la ley como el núcleo prioritario de la política exterior y de seguridad más allá del $2019^{14}$.

\section{Política comercial común y condicionalidad}

En el marco de la política comercial común (art. 207 TFUE), y también en la política de cooperación al desarrollo (art. 208 TFUE), la Unión ha utilizado diversos instrumentos en sus relaciones con terceros Estados a fin de promocionar y proteger los derechos humanos y reafirmar de este modo su identidad europea como garante del derecho internacional de los derechos humanos. El uso de la condicionalidad en estos instrumentos supedita la obtención de determinados beneficios al cumplimiento de una serie de requisitos previamente establecidos. En general, el Acuerdo persigue el objetivo de condicionar su cumplimiento a la observancia de unos estándares mínimos de protección de los derechos y libertades fundamentales y los principios democráticos. De este modo, la concesión de ayudas económicas a los países terceros con los que la UE establece relaciones comerciales o económicas está supeditada al cumplimiento de los estándares que la UE considera esenciales ${ }^{15}$. En el marco de las relaciones exteriores de la Unión, la introducción de estos mecanismos de condicionalidad a través de la polí-

13 Comunicación conjunta al Parlamento Europea y al Consejo. Plan de acción....6 y 7.

14 Doc. 12815/17, «Council Conclusions on the Mid-Term Review of the Action Plan on Human Rights and Democracy «, Luxembourg, 16 October 2017. Acceso el 12 de enero de 2018, http://www.consilium.europa.eu/media/21512/st12815

15 Lucas Rodríguez de las Heras, «La introducción de la cláusula democrática en la política exterior de la Unión Europea», en La acción exterior de la Unión Europea en materia de derechos humanos, coord. por Ana Manero Salvador (Madrid: Instituto Universitario de Estudios Internacionales y Europeos «Francisco de Vitoria», 2014): 93. 
tica comercial convierte esta dimensión en una extensión de esta acción exterior en materia de derechos humanos ${ }^{16}$.

La condicionalidad tiene dos vertientes, una negativa y otra positiva. La negativa comporta un «castigo», que implica la suspensión o la terminación de las relaciones con los países que violan los derechos humanos o vulneran la democracia. En cambio, la positiva es un incentivo para que los países mejoren el grado de cumplimiento de sus obligaciones, tanto en materia de derechos humanos como en relación a otras cuestiones vinculadas, como la gobernanza, la lucha contra la producción y el tráfico de drogas y la protección medioambiental. El cumplimiento de estos estándares permite mejorar el acceso de sus productos en el mercado europeo. La UE ha introducido estas cláusulas de condicionalidad en diversos tipos de acuerdos internacionales, de carácter comercial o económico, como los de cooperación o ayuda al desarrollo, y también en otro tipo de instrumentos comerciales de carácter unilateral, como por ejemplo, el instrumento de defensa comercial, el nuevo sistema de preferencias generalizadas (SPG), los códigos voluntarios de buena conducta y el etiquetado social voluntario ${ }^{17}$.

\section{Origen y tipología de las cláusulas de condicionalidad}

El origen de las cláusulas de condicionalidad se sitúa en las primeras relaciones comerciales de las Comunidades Europeas (CCEE) con sus ex colonias, a través de la firma de los tratados de ayuda al desarrollo. En los primeros Acuerdos de Yaundé ${ }^{18}$ se hacían referencias genéricas a los derechos humanos, aludiendo a los principios de Naciones Unidas, pero sin un efecto jurídico concreto ${ }^{19}$. Durante toda la década de 1970, aunque las CCEE empezaron a dictar las primeras condenas contra regímenes dictatoriales y los abu-

16 Ana Manero Salvador, «Las preferencias europeas y la condicionalidad: balance y perspectivas de futuro», en La acción exterior de la Unión Europea en materia de derechos humanos, coord. por Ana Manero Salvador (Madrid: Instituto Universitario de Estudios Internacionales y Europeos «Francisco de Vitoria», 2014), 103.

17 Teresa Fajardo del Castillo y Luis Miguel Hinojosa Martínez, «Los nuevos problemas del comercio internacional y la Ronda de Doha», en Derecho internacional económico, coord. por Luis Miguel Hinojosa Martínez y Javier Roldán Barbero (Madrid: Marcial Pons, 2010), 223-226.

${ }^{18}$ Acuerdo de Yaoundé I, de 20 de julio de 1963 (DOCE L 93, de 11 de junio de 1964); y Acuerdo de Yaoundé II, de 29 de julio de 1969 (DOCE 282, de 28 de diciembre de 1970).

19 Gerhard Niedrist, «Las cláusulas de derechos humanos en los tratados de libre comercio de la Unión Europea», Anuario Mexicano de Derecho Internacional XI, (2011): 467. Véase también Ana Gómez Consarnau, «El uso de la cláusula democrática y de derechos humanos en las relaciones exteriores de la Unión Europea», Institut Universitari d'Estudis Europeus, Observatori de Política Exterior Europea, Working Paper n. ${ }^{\circ} 39$, (2009): 1-4. 
sos cometidos sobre sus poblaciones, las CCEE continuaron manteniendo una estricta separación entre las cuestiones comerciales y económicas y cualquier posible consideración política. Por tanto, los derechos humanos y su protección estaban excluidos de cualquier tratado económico y comercial. El motivo no era sólo las reticencias de las instituciones comunitarias, sino también la desconfianza de los países receptores de esta política comercial, que entendían esta condicionalidad como un reflejo de posiciones imperialistas, eurocéntricas o una intrusión en sus políticas internas ${ }^{20}$. Los posteriores Acuerdos de Lomé I (1975-1979) y Lomé II (1979-1985) no incluyeron tampoco ninguna referencia al respeto de los derechos humanos, ni a la observancia de un estándar mínimo de protección. El cambio empezó a gestarse con los Acuerdos de Lomé III (1985-1990), momento en que se produce un giro en las circunstancias económicas que debilitó las posiciones mantenidas por los países ACP y, por el contrario, fortaleció las capacidades de las CCEE para introducir progresivamente las cuestiones de derechos humanos en los acuerdos. En este primer momento, la cláusula sólo es un fundamento político que inspira sus políticas nacionales e internacionales. Todavía no se configura como un elemento esencial del Acuerdo, cuya vulneración pueda comportar consecuencias jurídicas de ningún tipo ${ }^{21}$. A partir del IV Convenio de Lomé (19901995) se incorpora en estos acuerdos una cláusula para el respeto de los derechos humanos y la democracia, estableciéndose por primera vez y de un modo inequívoco una relación entre desarrollo y derechos humanos. Los derechos humanos pasan a ser un elemento fundamental, aunque no esencial del tratado, por lo que estas cláusulas todavía no eran suficiente base jurídica para poder suspender o denunciar los acuerdos en caso de violaciones graves de los derechos humanos o en caso de interrupción del proceso democrático ${ }^{22}$. Cuando en 1992 el Consejo de la UE decidió incluir en todos los acuerdos celebrados con los países vecinos europeos una cláusula que permitiese la suspensión o la toma de otras medidas en caso de violaciones a los derechos humanos y a los procesos democráticos, sí que aparece una nueva modalidad de cláusula, la del elemento esencial. Esta nueva cláusula autoriza de modo inequívoco a la toma de medidas en caso de violaciones graves de los derechos humanos. Así, desde 1995 las cláusulas de condicionalidad pasan a generalizarse en casi todos los acuerdos internacionales celebrados por las CCEE/ UE con terceros países, ya sean acuerdos comerciales bilaterales, acuerdos de

${ }^{20}$ Rodríguez de las Heras, «La introducción de la cláusula democrática en la política exterior de la Unión Europea», 93.

${ }^{21}$ M. a del Carmen Muñoz Rodríguez, Democracia y derechos humanos en la acción exterior de la Unión Europea, (Madrid: ed. Reus, 2011), 176.

22 Rodríguez de las Heras, «La introducción de la cláusula democrática en la política exterior de la Unión Europea», 91-100. 
colaboración y cooperación, o acuerdos de asociación ${ }^{23}$. El contenido de la cláusula recoge tanto la fórmula más extendida de respeto de la democracia y de los derechos humanos como elemento fundamental del acuerdo (cláusula de democracia y derechos humanos), como la llamada cláusula social, que persigue la abolición de prácticas odiosas como la esclavitud, el trabajo forzado u otras. Las diferentes fórmulas, que pueden clasificarse no sólo atendiendo a su contenido sino también a su aplicación geográfica o por países o incluso en virtud de su severidad, ponen de relieve la pluralidad de definiciones y la dificultad de la tipología de la condicionalidad política. La complejidad de clasificación se corresponde con una práctica llena de incoherencias en la que las consideraciones de Realpolitik tienen un peso esencial en la acción de la Unión ${ }^{24}$. En todo caso, la cláusula democrática y de derechos humanos y la cláusula social, se han convertido en una cláusula del elemento esencial que se ha generalizado en todos los acuerdos internacionales generales, de carácter económico o comercial.

\section{La cláusula democrática y de derechos humanos}

Esta cláusula fue introducida por primera vez como un elemento esencial de los acuerdos de la UE en el art. 9.1, párr. 2 del Acuerdo de Cotonou de 2000, otorgándole un valor jurídicamente vinculante. Su gran innovación es considerar como elementos esenciales del Acuerdo (con independencia de su tipología: económico, de partenariado o de cooperación) el respeto de los derechos fundamentales, ya se trate de derechos civiles o políticos, o de derechos económicos, sociales y culturales ${ }^{25}$. Ante el incumplimiento de este elemento estimado esencial se puede dar por terminado o bien proceder a la suspensión total o parcial del Acuerdo. La fundamentación jurídica de esta suspensión radica en el artículo 60 del Convenio de Viena sobre el Derecho de los Tratados, que otorga la obligatoriedad y la suficiente base jurídica para su aplicación ${ }^{26}$.

23 Alicia Cebada Romero, «La cláusula democracia/derechos humanos como instrumento de condicionalidad en las relaciones exteriores de la CE» en La Unión Europea ante el siglo XXI: los retos de Niza. Actas de las XIX Jornadas de la Asociación Española de Profesores de Derecho Internacional y Relaciones Internacionales, coord. por Concepción Escobar Hernández (Madrid: BOE, 2003), 89.

${ }^{24}$ Amaya Úbeda de Torres, «La evolución de la condicionalidad política en el seno de la Unión Europea», Revista de Derecho Comunitario Europeo 32, (2009): 68-69.

25 Art. 9.2 del Acuerdo de Cotonou, de 23 de junio de 2000 (DOUE L 317, de 15 de diciembre de 2000).

${ }^{26}$ Rodríguez de las Heras, «Introducción de la cláusula democrática en la política exterior de la Unión Europea», 96. 


\section{Otras formulaciones}

Parte de la doctrina considera que la cláusula del elemento esencial ha sido modificada y ampliada a través de fórmulas avanzadas, aunque para otros autores estaríamos ante cláusulas distintas. Dejando al margen este debate doctrinal, estaríamos hablando de las cláusulas conocidas como cláusula báltica y cláusula búlgara ${ }^{27}$.

A partir de 1992 los acuerdos celebrados con las Repúblicas Bálticas y con Albania incluían la denominada cláusula báltica, que supone una suspensión automática del Acuerdo de forma total o parcial, si se infringe un elemento esencial, lo que constituye una excepción al plazo de tres meses que exige la Convención de Viena sobre derecho de los tratados de 1969. Pero esta cláusula que fue rápidamente sustituida por la cláusula búlgara o de no ejecución — usada en 1993 en los acuerdos con Bulgaria - regula un procedimiento que permite evitar la suspensión o la terminación, que sólo sería posible como último recurso. En un primer momento, si no hay una urgencia especial - en cuyo caso sí se admite la adopción inmediata de medidas negativas - se debe intentar una conciliación entre las partes, en base al diálogo y el análisis de la situación. En una segunda fase, fracasada la conciliación, sí se impondrían las medidas apropiadas, sometidas a una graduación. Esta es la cláusula que se utilizó finalmente como fórmula estándar en todos los acuerdos con terceros países a partir de 1995 de modo sistemático $^{28}$, ya que según la UE no era tan «drástica» como la báltica y permitía la máxima «operatividad» de los acuerdos ${ }^{29}$. Encontramos esta cláusula en los acuerdos con Chile y MERCOSUR, que van además acompañados de una declaración interpretativa acerca de las alternativas a la suspensión o la terminación, así como una lista de lo que se considera que constituye una situación de urgencia. También fue incorporada en la versión revisada del Acuerdo de Lomé IV de 1995, así como en el Acuerdo que se ocupa ahora de las relaciones con los países ACP, firmado en Cotonou el 23 de junio de

27 Véase Úbeda de Torres, «La evolución de la condicionalidad política en el seno de la Unión Europea», 49-88.

${ }_{28}$ Comunicación de la Comisión sobre la inclusión del respeto de los principios democráticos y de los derechos humanos en acuerdos entre la CE y terceros Estados, COM (95) 216, de 23 de mayo de 1995.

29 Malena Zingoni-Fernández, La cláusula democrática y la Política exterior de la Unión Europea, 16. Acceso el 9 de enero de 2018, http://studylib.es/doc/257370/lacl\%C3\%A1usula-democr\%C3\%A1tica-y-de-derechos-humanos. Véase también, Parlamento Europeo, «Documento de trabajo sobre la cláusula de respeto de los derechos humanos y de los principios democráticos en los acuerdos de la Unión Europea», DT/577923ES.doc, de 23 de agosto de 2005. Acceso el 12 de febrero de 2018, http://www .europarl.europa.eu/ meetdocs/2004_2009/documents/dt/577/577923/577923es.pdf . 
2000 (vigente desde el 1 de abril de 2003). El Acuerdo fue revisado por primera vez en el 2005. En aras de mayor claridad, el art. 9 de este Acuerdo se renombró como «Elementos esenciales en materia de derechos humanos, principios democráticos y el Estado de Derecho y elemento fundamental en materia de buena gobernanza ${ }^{30}{ }_{\gg}$. De este modo, en caso de producirse una violación grave y persistente de los derechos humanos, y por lo tanto una vulneración de la cláusula, se permite la adopción de una serie de medidas para ajustarlas a cada circunstancia Ahora bien, en tanto que esta cláusula no está orientada a la punición sino al fomento del diálogo y la cooperación entre socios, la suspensión sólo se utiliza como último recurso. Igualmente, la aplicación o el levantamiento de sanciones no responden siempre a criterios objetivos. Ante la constatación de violaciones serias ya sea de los derechos humanos o de la interrupción del proceso democrático en uno de los países contemplados en el Acuerdo de Cotonou, la Unión promueve primero el diálogo político (por tanto, prima siempre el enfoque positivo) y, en caso de que este fracase, contemplará la imposición de medidas negativas de severidad creciente que sean plenamente respetuosas con el principio de proporcionalidad.

El análisis de la práctica de la UE en la aplicación de esta cláusula según regiones muestra que las sanciones a la ayuda han tenido mayor cabida en el África Subsahariana, donde los Estados miembros tienen poco que perder. En países como Turquía, Nigeria o China, tras observar graves abusos de los derechos humanos y reveses en los procesos democráticos, se han sucedido ejemplos de medidas sin fuerza o parciales; y en otros casos, como Argelia, Egipto, Colombia o Rusia, donde persisten violaciones de derechos humanos, no ha habido sanciones ${ }^{31}$. Esta cláusula ha sido invocada en doce ocasiones desde 1995 hasta el 2010 como base para consultas, suspensión de ayuda y otras medidas que han afectado a diez Estados ACP: Níger, Guinea-Bissau, República Centroafricana, Togo, Haití, Comoras, Costa de Marfil, Fiyi, Liberia y Zimbabue. El análisis demuestra que la falta de correlación entre el grado de violaciones de los derechos humanos y el nivel de sanción determina el fracaso en la aplicación de las políticas de derechos humanos sobre la base de criterios objetivos, como lo demos-

${ }^{30}$ Decisión 2005/599/CEE del Consejo, de 21 de junio de 2005, y texto del Acuerdo (DOL 209/26, de 11 de agosto de 2005). La segunda revisión se produjo en el 2010, por medio de la decisión 2010/648/UE, del Consejo de 14 de mayo de 2010 (DO L 287/1, de 4.11.2010) para adaptarlo a las modificaciones tratado de Lisboa.Véase también, Cintia DíazSilveira Santos, «La cláusula de derechos humanos y democráticos en las relaciones entre la UE y América latina y el Caribe», Revista Electrónica Iberoamericana 1, n. ${ }^{\circ} .1$ (2007): 103.

31 Angela Ward, «Frameworks for cooperation between the European Union and third states: a viable matrix uniform human rights standards? European Foreign Affairs Review 3, (1998): 154. 
traría la reanudación parcial de la cooperación con Sudán en enero de 2005, a pesar de la persistencia de graves violaciones de los derechos humanos en la región de Darfur ${ }^{32}$.

De aquí se concluye que a pesar de la evolución importante en la construcción de la condicionalidad no existe un mandato jurídico directo por el que sea obligatoria la inserción de las cláusulas de democracia y derechos humanos, y ello se refleja en la variedad de fórmulas y modelos seguidos. Aunque está claro el uso de la condicionalidad por parte de la UE en sus relaciones comerciales con terceros países, la práctica demuestra que tiene enfrente el reto de su aplicación coherente, no siempre conseguida, puesto que, por un lado, la aplicación de esta cláusula de condicionalidad política implica vencer la apatía de los Estados a cuestionar la validez del principio de no intervención; y, de otro lado, la aplicación de la condicionalidad está sometida a consideraciones subjetivas, de carácter político y económico que a menudo priman sobre las consideraciones jurídicas ${ }^{33}$.

\section{Efectos jurídicos del incumplimiento de la de la cláusula democrática}

Como ya hemos apuntado, la cláusula democrática, convertida en un elemento esencial del Acuerdo, permite la suspensión o finalización del Acuerdo cuando una de las Partes considere que la otra ha violado de forma grave los derechos humanos o los procesos democráticos, aunque no de un modo automático. El incumplimiento de una obligación derivada del respeto a esta cláusula dará lugar a la celebración de consultas para intentar remediar la situación y si estas no conducen a una solución aceptable se adoptarán las medidas pertinentes. Estas medidas deben adoptarse de conformidad con el Derecho internacional y ser proporcionales a la violación alegada por una de las Partes, siendo prioritario elegir aquellas medidas que no perturben la aplicación el Acuerdo. Por este motivo, la suspensión del Acuerdo es el último recurso ${ }^{34}$. Las medidas a adoptar están recogidas en el Anexo 2 de la Comunicación de 1995, y tienen distintos grados que van desde la modificación o reducción de la cooperación hasta su suspensión.

La práctica de la UE demuestra que no ha habido prácticamente ninguna suspensión de acuerdos invocando el incumplimiento de esta cláusula, de hecho la UE continúa otorgando concesiones comerciales y ayudas

32 Díaz-Silveira Santos, «La cláusula de derechos humanos y democráticos en las relaciones entre la UE y América latina y el Caribe», 104-105.

33 Úbeda de Torres, «La evolución de la condicionalidad política en el seno de la Unión Europea», 72 .

34 Art. 96.2, literales a), b) y c) del Acuerdo de Cotonou. 
económicas al Estado infractor ${ }^{35}$. Una explicación se basaría en que esta cláusula, si bien se incluye de forma sistemática en los acuerdos generales, no ocurre lo mismo con su incorporación en los acuerdos sectoriales ${ }^{36}$. De modo que si la relación no se basa en un acuerdo de asociación o de cooperación, la UE no impone las exigencias al respeto de esta cláusula. Y además, sólo en aquellos acuerdos de países geográfica e históricamente próximos, o con los económicamente más débiles se ha mantenido de manera general este tipo de cláusulas y se han llevado a cabo suspensiones de los acuerdos ${ }^{37}$. En cambio, respecto a los países candidatos a la adhesión, se ha puesto en marcha un estricto mecanismo de condicionalidad. Asimismo, en la Política Europea de Vecindad renovada, se ha integrado un enfoque más por más (más integración y fondos a cambio de más reformas). A su vez, en las preferencias comerciales concedidas por la Unión a los países en desarrollo (SPG+) se prevén incentivos a las reformas.

\section{Instrumentos comerciales unilaterales y condicionalidad}

También en relación con los derechos sociales la Unión defiende la vinculación entre las normas internacionales del trabajo y el derecho internacional del comercio. Así, desde la década de los noventa, la UE ha adoptado distintos instrumentos de condicionalidad social, en este caso negativa, como es el instrumento de defensa comercial ${ }^{38}$ y los códigos voluntarios

${ }^{35}$ Un ejemplo es el Tratado de Libre comercio UE-Colombia-Perú (2010) que deja mucho que desear en materia derechos humanos y estándares ambientales y socio laborales. Sólo el art. 1 contiene una referencia en materia de derechos humanos al incorporar la «cláusula democrática» por remisión al Acuerdo de diálogo político y cooperación de 2003 entre la Comunidad Andina de Naciones (CAN) y la UE. Véase Jaume Saura Estapà,»Implicaciones de derechos humanos en el tratado de libre comercio entre Colombia y la Unión Europea», InDret 4, (Barcelona, 2013): 1-25.

${ }^{36}$ Ana Gómez Consarnau, «El uso de la cláusula democrática y de derechos humanos en las relaciones exteriores de la Unión Europea», Observatori de Política Exterior Europea, IUEE, working paper núm. 39 (2009), 8. Acceso el 09 de enero de 2018, http://www.recercat. cat/bitstream/handle/2072/204341/N.\%2039.pdf?sequence $=1$.

37 Rodríguez de las Heras, «La introducción de la cláusula democrática en la política exterior de la Unión Europea», 99-100. En relación con los países ACP, véase Niedrist, «Las cláusulas de derechos humanos en los tratados de libre comercio de la Unión Europea», 478-480.

38 Reglamento (CE) n. ${ }^{\circ}$ 3286/94 del Consejo ((DOUE L 349, de 31 de diciembre de 1994). El reglamento fue modificado el 15 de enero de 2014 por el Reglamento (CE) n. ${ }^{\circ}$ 37/2014 2014 del Consejo, de 12 de febrero de 2008, sobre el establecimiento de procedimientos comunitarios en el ámbito de la política comercial común (DOUE L 18, de 21 de enero de 2014). Véase Luis Miguel Hinojosa Martínez, Comercio justo y derechos sociales, (Madrid: Tecnos, 2002), 126. 
de buena conducta y etiquetado social ${ }^{39}$. En todo caso, tanto el código voluntario de buena conducta como el etiquetado social son mecanismos que adolecen de problemas técnicos y jurídicos, relacionados con la falta de seguridad jurídica, puesto que a menudo los estándares sociales aplicados no guardan relación con los derechos y libertades fundamentales en el trabajo proclamados por la OIT. Y además, la validez del etiquetaje sigue condicionada al cumplimiento de las normas de la $\mathrm{OMC}^{40}$. Por ello nos centraremos en el instrumento comercial de condicionalidad social positiva más desarrollado por la UE hasta el momento: el nuevo sistema de preferencias generalizadas (SPG).

\section{La condicionalidad social y el nuevo sistema de preferencias generalizadas}

Aunque en sus inicios las medidas de condicionalidad perseguían, - a través de la celebración de acuerdos comerciales con terceros países-, cambios en los sectores económicos del Estado receptor de la ayuda, la UE empezó también a utilizarlas, de modo unilateral, en sus políticas de desarrollo e introducir así la promoción y protección de los derechos humanos y los principios democráticos como uno de sus ejes fundamentales en su acción exterior. En este sentido, la medida comercial de más «éxito» utilizada en las relaciones exteriores de la UE ha sido el SPG, por lo que es necesario analizar la evolución de la condicionalidad positiva en este sistema y los cambios a los que se enfrenta con la nueva regulación que comenzó a aplicarse el 1 de enero de 2014.

Desde 1971 la UE aplica un SPG en sus relaciones comerciales con los países en vías de desarrollo (PVD), a fin de prestarles asistencia en su

39 Aunque se adoptan mayoritariamente en el ámbito privado de las empresas, algunas organizaciones internacionales, en especial la OIT y la OCDE, han empezado a adoptarlos. Véase en general Janelle Diller, «¿Una conciencia social en el mercado mundial? Dimensiones laborales de los códigos de conducta, el etiquetado social y las iniciativas de los inversores», Revista Internacional del Trabajo 118, n. ${ }^{\circ} .2$, (1999): 111-145, doi:10.1111/ j.1564-913X.1999.tb00069.x. Y también, Resolución (UE) n. ${ }^{\circ}$ A4-0508/98, del Parlamento Europeo, sobre la adopción de normas por la Unión Europea para las empresas europeas que operan en países en vías de desarrollo: hacia un código de conducta europeo (DOUE C 104, de 14 de abril de 1999); y Comisión Europea, Libro verde: Fomentar un marco europeo para la responsabilidad social de las empresas, COM/2001/0366 final, Bruselas, 18 de julio de 2001.

40 Víctor M. Sánchez, «Globalització i protecció internacional dels drets socials», en Els drets humans al segle XXI: continuïtat $i$ canvis, dir. por Jordi Bonet Pérez y Víctor M. Sánchez, (Barcelona: Huygens, 2007), 424. 
lucha contra la pobreza y ayudarles a generar ingresos a través del comercio internacional. Este SPG ha sufrido una interesante evolución ${ }^{41}$, dado que siguiendo la tendencia a la introducción de disposiciones relativas al respeto por la democracia y los derechos humanos en los acuerdos con terceros países, la UE, a través de las preferencias generalizadas, ha introducido incentivos para mejorar el cumplimiento de determinadas obligaciones internacionales relativas a los derechos humanos, incluidos los derechos económicos, sociales y culturales.

En 1995 se produce un cambio de paradigma en el SPG, con la inclusión de mecanismo de condicionalidad positiva. La UE ofrece, sin esperar reciprocidad a cambio, la reducción de aranceles o el acceso en franquicia de derechos al mercado de la Unión, así como beneficios especiales a determinados países menos desarrollados y a aquellos países que cumplan determinadas normas básicas en materia laboral y de medio ambiente ${ }^{42}$. Por primera vez se establecen preferencias adicionales para aquellos países en desarrollo que cumplan con estándares laborales (cláusula social). Estas preferencias se completaron en 2001 con el régimen de droga y medioambiental, así como con la inclusión del régimen específico para los países menos avanzados, Todo menos armas $^{43}$.

La Comunicación de la Comisión Europea de 7 de julio de 2004 sobre el $\mathrm{SPG}^{44}$ abogaba por un sistema de más fácil acceso para los operadores, reducir el número de regímenes establecidos en cinco, y centrarse en los países que más lo necesitaran, fomentando diversos modos la cooperación regional entre los países en desarrollo. Así, el nuevo SPG debería ayudar a esos países a alcanzar un nivel de competitividad que los convirtiera en económicamente autosuficientes y en socios comerciales de pleno derecho.

41 Joan David Janer Torrens, «Sistema de preferencias generalizadas y promoción de los derechos humanos en la acción exterior de la Unión Europea», en Retos para la acción exterior de la Unión Europea, dir. por Carmen Martínez Capdevila y Enrique J. Martínez Pérez (Tirant lo blanch: Valencia, 2017), 639-646.

${ }^{42}$ Comisión Europea, DG de Comercio, «El sistema de preferencias generalizadas de la Unión Europea», Bélgica, febrero de 2004. Acceso el 12 de febrero de 2018, http://trade. ec.europa.eu/doclib/docs/2004/march/tradoc_116449.pdf .

${ }^{43}$ Manero Salvador, «Las preferencias europeas y la condicionalidad: balance y perspectivas de futuro», 103-104.

${ }^{44}$ Comunicación de la Comisión de 7 de julio de 2004, «Países en desarrollo, comercio internacional y desarrollo sostenible: la función del sistema de preferencias generalizadas (SPG) de la Comunidad para el decenio 2006/2015», COM (2004), 461 final, Bruselas, 7 de julio de 2004 . 


\section{Régimen general y régimen específico}

A partir de las sugerencias de la Comisión, el Parlamento y el Consejo adoptaron el Reglamento 978/2012 45 - vigente desde el 1 de enero de 2014 para los próximos diez años - que diseña un sistema general de preferencias arancelarias (arts. 4 a 8 ) y dos regímenes específicos: a) un régimen especial de estímulo al desarrollo sostenible y la gobernanza (SPG+) (arts. 9 a 16), y b) un régimen especial en favor de los países menos desarrollados (arts. 17 a 18). Debemos destacar el régimen especial de estímulo (SPG+), que se basa en el concepto integral de desarrollo sostenible reconocido en convenios e instrumentos internacionales diversos, y tiene por objeto ofrecer preferencias arancelarias adicionales a aquellos países en vías de desarrollo, con el fin de erradicar la pobreza. De este modo se hace presente la condicionalidad positiva en el SPG. Los requisitos que deben cumplir los países solicitantes para beneficiarse de estas preferencias están establecidos en el art.9.1: a) Tener la consideración de «vulnerables», como consecuencia de su falta de diversificación y de su insuficiente integración en el sistema de comercio internacional, según la definición establecida en el anexo VII del reglamento; b) Haber ratificado los convenios enumerados en el anexo VIII del reglamento (los convenios pertinentes) y, de acuerdo con las últimas conclusiones disponibles de los organismos de seguimiento del cumplimiento de estos convenios, no conste ninguna falta grave a la obligación de ejecutar los convenios» ${ }^{46}$; c) No haber formulado ninguna reserva

45 Reglamento (UE) n. ${ }^{\circ}$ 978/2012, del Parlamento Europeo y del Consejo, de 25 de octubre de 2012, por el que se aplica un sistema de preferencias arancelarias generalizadas y se deroga el Reglamento (CE) n. ${ }^{\circ} 732 / 2008$ del Consejo (DOUE L 303, de 31 de octubre de 2012).

${ }^{46}$ Los principales convenios internacionales sobre derechos humanos y laborales de las Naciones Unidas y de la OIT a los que se remite el art. 9 en el anexo son: La Convención para la Prevención y la Sanción del Delito de Genocidio (1948); la Convención Internacional sobre la Eliminación de todas las Formas de Discriminación Racial (1965); el Pacto Internacional de Derechos Civiles y Políticos (1966); el Pacto Internacional de Derechos Económicos, Sociales y Culturales (1966); la Convención sobre la Eliminación de todas las Formas de Discriminación contra la Mujer (1979); la Convención contra la Tortura y Otros Tratos o Penas Crueles, Inhumanos o Degradantes (1984); la Convención sobre los Derechos del Niño (1989); el Convenio sobre el Trabajo Forzoso, n. ${ }^{2} 29$ (1930); el Convenio sobre la Libertad Sindical y la Protección del Derecho de Sindicación, n. ${ }^{\circ} 87$ (1948); el Convenio sobre el Derecho de Sindicación y de Negociación Colectiva, n. ${ }^{\circ} 98$ (1949); el Convenio relativo a la Igualdad de Remuneración entre la Mano de Obra Masculina y la Mano de Obra Femenina por un Trabajo de Igual Valor, n. ${ }^{\circ} 100$ (1951); el Convenio sobre la Abolición del Trabajo Forzoso, n. ${ }^{\circ} 105$ (1957); el Convenio relativo a la Discriminación en Materia de Empleo y Ocupación, n. ${ }^{\circ} 111$ (1958); el Convenio sobre la Edad Mínima, n. ${ }^{\circ} 138$ (1973); y el Convenio sobre la Prohibición de las Peores Formas de Trabajo Infantil y la Acción Inmediata para su Eliminación, n. ${ }^{\circ} 182$ (1999)». 
en relación con los convenios pertinentes que esté prohibida por los convenios pertinentes, o sea considerada por el art. 9 como incompatible con el objetico y finalidad del convenio; d) Asumir el compromiso vinculante de mantener la ratificación de los convenios pertinentes y garantizar su aplicación efectiva; e) Aceptar sin reservas los requisitos de información impuestos por cada convenio y asumir el compromiso vinculante de aceptar el seguimiento y la revisión regulares de su historial de aplicación, de acuerdo con lo que dispongan los convenios pertinentes; y f) Asumir el compromiso vinculante de participar en el procedimiento de seguimiento establecido en el art. 13.

\section{Procedimiento de seguimiento}

El reglamento también establece un sistema de seguimiento por el que faculta a la Comisión Europea para que verifique el estado de la ratificación de los convenios internacionales sobre derechos humanos y laborales y su aplicación efectiva, así como presentar un informe, cada dos años al Parlamento y al Consejo sobre el estado de ratificación y de cumplimiento de estos convenios, según la información aportada por los países beneficiarios, que están obligados a proporcionarla (art.13 y 14).

En el caso de «violaciones graves y sistemáticas» de los principios establecidos en algunos convenios internacionales relativos a derechos humanos y laborales, el reglamento prevé la retirada temporal de las preferencias (art. 15.1). Esta retirada de las preferencias, ya sea total o parcial de los productos provenientes de un país beneficiario se limita a tres supuestos (art. 13 y 14): a) No respetar el compromiso vinculante de mantener la ratificación y la aplicación efectiva de dichos convenios, según establece el art.9; b) Formular una reserva prohibida por los convenios pertinentes o incompatible con los objetivos y finalidad de los respectivos convenios, y c) No respetar en la práctica los compromisos vinculantes de mantener la ratificación, las garantías de aplicación, los requisitos de información y la participación en el procedimiento de seguimiento.

La carga de la prueba del cumplimiento de las obligaciones del art. 9 corresponde al país beneficiario y la Comisión puede suspender las preferencias a partir de la duda razonable, que se deriva de las conclusiones del informe que elabora o de las pruebas disponibles, previa información al Parlamento Europeo y al Consejo ${ }^{47}$. Por lo tanto, el incumplimiento de los

${ }^{47}$ Esta retirada temporal se publicará en el DOUE y se comunicará al país afectado (art. 14.3). 
convenios recogidos en la Parte A del anexo VIII, puede dar pie a la retirada temporal, total o parcial de las preferencias a los productos de los países beneficiarios ${ }^{48}$.

\section{Práctica de la Unión Europea}

La práctica de la UE muestra que la regulación de este régimen de estímulo específico sometido a la condicionalidad positiva favorece la protección de determinados estándares laborales básicos, que se corresponden con los contenidos básicos de los derechos fundamentales de la OIT. Si bien su infracción no impide que el país beneficiario pueda seguir beneficiándose del régimen general de las preferencias. Antes de la vigencia de este nuevo sistema, en la década de los noventa del siglo pasado la UE ya se planteó la retirada temporal de las preferencias arancelarias a Pakistán, India, China y Rusia, aunque no se llevaron a cabo ${ }^{49}$. En la actualidad, el mecanismo de seguimiento sólo se ha aplicado con Sri Lanka, Bielorrusia y Myanmar, a pesar de que en la lista de receptores de preferencias figuran países que se caracterizan por su falta de respeto, incluso de interés por los derechos humanos ${ }^{50}$. En todo caso, sí es de destacar que para la UE es suficiente con probar de modo general la violación de derechos en el país concernido, sin que sea necesario demostrar que la práctica en cuestión afecte específicamente a los productos importados en el mercado de la Unión ${ }^{51}$. Aunque también es cierto que a menudo estas suspensiones son temporales y los países beneficiarios continúan disfrutando de este régimen ${ }^{52}$.

48 La Comisión adoptó el Reglamento (UE) delegado n. ${ }^{\circ}$ 1083/2013, de la Comisión, de 28 de agosto (DOUE L 293, de 5 de noviembre de 2013), para dar mayor transparencia y seguridad en el procedimiento de retirada tanto de los regímenes especiales como del general, detallando los plazos, los derechos de las partes y la revisión de la suspensión decretada.

49 Janer Torrens, «Sistema de preferencias generalizadas y promoción de los derechos humanos en la acción exterior de la Unión Europea», 650.

50 Por ejemplo Honduras, que a pesar de poner en entredicho el cumplimiento de los convenios a los que obliga el reglamento, sigue beneficiándose del SPG+. Véase Manero Salvador, «Las preferencias europeas y la condicionalidad: balance y perspectivas de futuro», 117.

51 Véase el caso de la retirada temporal de las preferencias concedidas a Myanmar, por las prácticas de trabajo forzado. Reglamento (CE) n. ${ }^{\circ}$ 552/97/CE, del Consejo, de 24 de marzo de 1997, por el que retira temporalmente a la Unión de Myanmar el beneficio de las preferencias arancelarias generalizadas (DO L85/8, de 27.3.1997).

52 Es lo que sucedió antes de la entrada en vigor del nuevo reglamento 978/2012 con El Salvador, Bielorrusia y Sri Lanka. Véase, Manero Salvador, «Las preferencias europeas y la condicionalidad...», 104, 106 y 107. 


\section{Reflexiones finales}

El cumplimiento de las disposiciones de los Tratados de la UE sobre derechos humanos - así como el derecho internacional de los derechos humanos - en sus relaciones con terceros países, ha guiado a la Unión hacia la construcción de una identidad europea que parece mostrar cierta unificación desde el año 2012 con la adopción del Marco estratégico sobre derechos humanos y democracia. Con este documento, la Unión muestra su preocupación por observar y promover de modo coherente los derechos humanos en su actuación exterior con el despliegue de sus políticas comunes. En el marco de la política comercial (y también de cooperación y ayuda al desarrollo), la construcción de esta identidad se ha basado en la adopción de un conjunto de instrumentos de carácter comercial, de entre los cuales la cláusula democrática y de derechos humanos y el Sistema de Preferencia Generalizadas (SPG) son los más utilizados en sus relaciones comerciales. Estos instrumentos priorizan la condicionalidad positiva por encima de la negativa, como vía para garantizar el cumplimiento de los derechos humanos en los países con los que la UE mantiene relaciones comerciales. A pesar de ello, existen un conjunto de problemas técnico-jurídicos y de voluntad política que hacen que no siempre se garantice el respeto a estos derechos y que tampoco sea fácil el control y seguimiento de su cumplimiento, lo que puede erosionar los efectos perseguidos.

La cláusula democrática y de derechos humanos está presente en la mayoría de acuerdos comerciales de carácter general, - pero no en los sectoriales que son mayoritarios-, puede mutar en un principio únicamente declarativo si no se crean mecanismos que permitan seguir la situación de cumplimiento de los derechos humanos. La práctica comercial de la UE demuestra que no ha habido prácticamente ninguna suspensión de acuerdos invocando el incumplimiento de esta cláusula, ni tampoco se han paralizado las concesiones comerciales ni las ayudas económicas, ya sea por falta de voluntad política o por desinterés de los países afectados.

En relación con el $S P G$, la Unión dispone por primera vez de un mecanismo jurídico que permite vincular la liberalización del comercio internacional con la protección de los derechos humanos en los países beneficiarios. Es probablemente el instrumento unilateral de carácter comercial que permite más claramente hacer efectivo el mandato de los Tratados a la Unión de promover y respetar los derechos humanos en su acción exterior. A pesar de ser atractivo para los países en vías de desarrollo a fin de facilitar su integración en el comercio internacional, también presenta problemas al centrarse en países que a menudo no respetan o no muestran interés en hacer cumplir los contenidos básicos de los derechos fundamentales de los convenios internacionales. Ello es debido a que la mayoría son países en 
vías de desarrollo y, por lo tanto, con mayores dificultades -o incluso desinterés - para cumplir con los convenios internacionales sobre derechos humanos. Igualmente existen problemas de índole técnico-jurídica que dificultan el seguimiento del cumplimiento por parte de estos países de los convenios internacionales. El procedimiento de seguimiento establecido es complejo y burocratizado, lo que puede fácilmente desincentivar a los países beneficiarios de este régimen a cumplir con sus obligaciones internacionales. Y más teniendo en cuenta la entidad de las reducciones arancelarias acordadas, a causa del descenso generalizado vía OMC de los aranceles a nivel mundial. Además, está sometido también a la intervención de otras organizaciones internacionales que deben verificar el cumplimiento de los convenios sobre derechos humanos por parte de los países terceros. Ello dificulta el control y la eficiencia del procedimiento. Por lo que sería deseable adoptar un marco normativo internacional que permita a la UE aplicar la condicionalidad social de modo general en sus relaciones comerciales con terceros. Así se evitaría la arbitrariedad y el proteccionismo que a menudo supone la aplicación de medidas unilaterales, y quizás solventaría las dificultades en compaginar la aplicación adecuada de los mecanismos de control de la Unión con los de los convenios internacionales.

En todo caso, la condicionalidad presente en el SPG y en la cláusula de democracia y derechos humanos pone en evidencia que la Unión ha optado por una actuación proactiva y no reactiva en sus relaciones con terceros países, en las que el comercio se convierte en el incentivo para fomentar el respeto y la promoción de los derechos humanos en el exterior.

\section{Sobre la autora}

Doctora en Sociedad de la información y la comunicación (especialidad Derecho internacional público y de la Unión Europea). Licenciada en Derecho y Máster en Estudios Europeos por el Insituto Universitario de Estudios Europeos de la UAB. Actualmente es profesora de derecho internacional público y de la UE en la Universitat Oberta de Catalunya (UOC) y Directora del Grado de Gestión y Administración Pública (interuniversitario: UOC,UB). Sus líneas de investigación principales son las operaciones de mantenimiento de la paz de la UE y la Política Común de Seguridad y defensa, y las relaciones exteriores de la UE y la promoción de los derechos humanos. Ha obtenido la acreditación de professor lector de la AQU (2015) y un sexenio de investigación de la AQU (2017). Es autora de una monografia sobre Las misiones de paz de la Unión Europea, Tirant lo Blanch, Valencia, 2015, 590 pág., y entre otros trabajos, ha publicado: «El factor tiempo en el proceso de planificación, creación y despliegue de las misiones 
de paz de la Unión Europea», REDI, Vol. 67, 2015, Madrid, pp. 123-155; «El Estatuto jurídico de las misiones de paz de la Unión Europea y la responsabilidad internacional por los daños causados en su actuación», $R G D E$, núm. 38, 2016, pp. 108-148.

\section{About the autor}

$\mathrm{PhD}$ in Information and Communication Society (specialty Public international law and European Union). Degree in Law and Master in European Studies from the University Institute of European Studies of the UAB. She is currently a professor of public international and EU law at the Open University of Catalonia (UOC) and Director of the Degree in Management and Public Administration (inter-university: UOC, UB). Its main lines of research are the EU peacekeeping operations and the Common Security and Defense Policy, and the EU's external relations and the promotion of human rights. She obtained the accreditation of reading professor (AQU) 2015 and she has awared a six-year investigation (AQU) 2017. She is the author of Peace Missions of the European Union, Tirant lo Blanch, Valencia, 2015, 590 p., and, among other works, she has published the following articles: «The time factor in the process of planning, creation and deployment of Peace missions of the European Union «, REDI, Vol. 67, 2015, Madrid, pp. 123-155; «The Legal Statute of European Union Peacekeeping Missions and International Liability for the Damages Caused by Their Action,» RGDE, no. 38, 2016, pp. 108-148. 


\section{Derechos de autor}

Los derechos de autor (para la distribución, comunicación pública, reproducción e inclusión en bases de datos de indexación y repositorios institucionales) de esta publicación (Cuadernos Europeos de Deusto, CED) pertenecen a la editorial Universidad de Deusto. El acceso al contenido digital de cualquier número de Cuadernos Europeos de Deusto es gratuito inmediatamente después de su publicación. Los trabajos podrán leerse, descargarse, copiar y difundir en cualquier medio sin fines comerciales y según lo previsto por la ley; sin la previa autorización de la Editorial (Universidad de Deusto) o el autor. Así mismo, los trabajos editados en CED pueden ser publicados con posterioridad en otros medios o revistas, siempre que el autor indique con claridad y en la primera nota a pie de página que el trabajo se publicó por primera vez en CED, con indicación del número, año, páginas y DOI (si procede). Cualquier otro uso de su contenido en cualquier medio o formato, ahora conocido o desarrollado en el futuro, requiere el permiso previo por escrito del titular de los derechos de autor.

\section{Copyright}

Copyright (for distribution, public communication, reproduction and inclusion in indexation databases and institutional repositories) of this publication (Cuadernos Europeos de Deusto, CED) belongs to the publisher University of Deusto. Access to the digital content of any Issue of Cuadernos Europeos de Deusto is free upon its publication. The content can be read, downloaded, copied, and distributed freely in any medium only for non-commercial purposes and in accordance with any applicable copyright legislation, without prior permission from the copyright holder (University of Deusto) or the author. Thus, the content of CED can be subsequently published in other media or journals, as long as the author clearly indicates in the first footnote that the work was published in CED for the first time, indicating the Issue number, year, pages, and DOI (if applicable). Any other use of its content in any medium or format, now known or developed in the future, requires prior written permission of the copyright holder. 